\title{
Composition and Fluorescence of Gadolinium (III) Acetyl- acetonate Derivatives by Solvothermal Method
}

\author{
Fengzhu Lv*, Yihe Zhang*, Xue Chen and Yong Ma
} Beijing Key Laboratory of Materials Utilization of Nonmetallic Minerals and Solid Wastes, National Laboratory of
Mineral Materials, School of Materials Science and Technology, China University of Geosciences, Beijing, China

\begin{abstract}
Gadolinium (III) acetylacetonate was treated by solvothermal method to produce gadolinium complexes with acetylacetonate and $-\mathrm{OH}$ groups. The composition, fluorescence, and magnetic properties of the products were studied in detail. The fluorescence showed the products possessed higher energy emission and excitation fluorescence compared to Gadolinium (III) acetylacetonate. Also the product produced at $160{ }^{\circ} \mathrm{C}$ possessed higher fluorescent intensity and responding ability toward external magnetic field. So the products were hydrophobic materials with magnetic and fluorescent properties.
\end{abstract}

Keywords

Gadolinium (III) acetylacetonate derivatives, Solvothermal method, Luminescence

\section{Introduction}

Generally, rare earth complexes have many potential applications in novel lighting devices, optical fibers and luminescent sensors benefiting from their long emission lifetime, narrow bandwidth and large Stokes shift in visible and near-infrared regions under Ultraviolet (UV) excitation [1-3]. The luminescence intensity of rare earth complexes related to the absorption efficiency of ligands, which is dependent on the ligand molecule with large $\pi$-conjugated system. Good solubility of substituted rare earth $\beta$-diketonate complexes in common organic solvents highly enhances their applications in the solution processable organic semiconductors and also provides more efficient approach to fabricate OLED devices by solution techniques $[4,5]$. So the fluorescence of some rare earth $\beta$-diketonate complexes were studied and modified to widen their application [6,7]. But preparation of multifunctional materials, such as materials with fluorescence and magnetic properties, by simple method is the project of researchers $[8,9]$.

Thermal decomposition of metal acetylacetonates has recently attracted much attention to fabricate thin films and nanoparticles of metals, metal alloys and metal oxides for microelectronic, catalytic and biomedical applications [1013]. Colloidal cubic $\mathrm{Gd}_{2} \mathrm{O}_{3}$ nanorings and nanoplates were selectively prepared from low-temperature $\left(90^{\circ} \mathrm{C}\right)$ hydrolysis of Gd-(AcAc) ${ }_{3}$ and subsequent in situ thermal dehydration of the hydrolyzed precursor-surfactant aggregates at $320^{\circ} \mathrm{C}$ [14]. But seldom reports focus on the partial decomposed samples as which is preferred possessing solubility in organic solvent and changed fluorescence compared to rare earth acetylacetonate.

In the present work Gadolinium (III) acetylacetonate hydrate was treated by solvothermal method in absolute diphenyl ether which is a water lack system where the hydrolysis of acetylacetonate is limited. The products

*Corresponding author: Fengzhu Lv and Yihe Zhang, Beijing Key Laboratory of Materials Utilization of Nonmetallic Minerals and Solid Wastes, National Laboratory of Mineral Materials, School of Materials Science and Technology, China University of Geosciences, No. 29 Xueyuan Road, Haidian District, Beijing 100083, China, Tel: +86-1082322759, Fax: +86-10-82322345, E-mail: Ifz619@cugb.edu.cn; zyh@cugb.edu.cn

Received: March 09, 2017: Accepted: July 22, 2017: Published: July 24, 2017

Copyright: (c) 2017 Lv F, et al. This is an open-access article distributed under the terms of the Creative Commons Attribution License, which permits unrestricted use, distribution, and reproduction in any medium, provided the original author and source are credited. 
were preferred possessing solubility in organic solvent and changed fluorescence compared to rare each acetylacetonate as well as magnetic properties. So different kinds of Gadolinium (III) acetylacetonate derivatives were produced. The composition, fluorescence and magnetic properties were studied in detail. Under appropriate preparation conditions, Gadolinium (III) acetylacetonate derivatives with higher fluorescent intensity and magnetic properties were produced.

\section{Experimental}

\section{Materials}

The raw material for synthesis of Gadolinium (III) acetylacetonate derivatives was gadolinium acetylacetonate trihydrate $\left(\mathrm{Gd}(\mathrm{AcAc})_{3} \cdot 3 \mathrm{H}_{2} \mathrm{O}, \mathrm{MW}=508.62 \mathrm{~g} /\right.$ mol), which was purchased from Beijing HWRK Chemical Co., Ltd.. Diphenyl ether was applied by Sinopharm Chemical Reagent Beijing Co., Ltd. Other solvents were from Beijing Chemical factory.

\section{Preparation of Gadolinium (III) acetylacetonate derivatives}

In a typical synthesis for the derivatives of $\mathrm{Gd}(\mathrm{A}$ cAc) $)_{3} \cdot 3 \mathrm{H}_{2} \mathrm{O}, 1.5787 \mathrm{~g}(0.003 \mathrm{~mol})$ of $\mathrm{Gd}(\mathrm{AcAc})_{3} \cdot 3 \mathrm{H}_{2} \mathrm{O}$ was dispersed into $10.5 \mathrm{~mL}$ of absolute diphenyl ether to form a solution under magnetic stirring, which was poured into $25 \mathrm{ml}$ teflon-lined autoclave and heated at different temperature for $2.0 \mathrm{~h}$. After that the autoclave was cooled to room temperature, the as-obtained yellow suspension was mixed with an equal volume of acetone, and the formed precipitate was separated by centrifugation and washed with acetone. The final samples were assigned as Gd-S-T, where T indicated the formed temperature by solvothermal. Gd-S-160 was grounded and calcinated at $450{ }^{\circ} \mathrm{C}$ for $10 \mathrm{~h}$ in air to give Gd-C- 160 . Afterwards, Gd-C-160 was furnace-cooled to room temperature, and grounded again into powder for measurement.

\section{Characterization}

Thermal Gravimetric Analysis (TGA) was measured on Perkin-Elmer TGA 7 with a heating rate of $20^{\circ} \mathrm{C} / \mathrm{min}$ ranging from $45^{\circ} \mathrm{C}$ to $800{ }^{\circ} \mathrm{C}$ in air, on the same time a NETZSCH STA 449F3 Thermo Gravimetric Analyser was coupled, using a heated capillary at $190{ }^{\circ} \mathrm{C}$, to a QMS 403 Mass Spectrometer (TGA-Ms) to identify the released gases during the thermal degradation of a material. The Fourier Transform Infrared Spectra (FTIR) of the solid samples was obtained with a Perkin Elmer Spectrum 100 FT-IR spectrometer. KBr pellet was used as background. The structure of the products was studied by powder X-ray diffraction (Rigaku D/max-rA) equipped with $\mathrm{Cu} \mathrm{Ka}(\lambda=0.1542 \mathrm{~nm})$ radiation at $40 \mathrm{kV}$ and $30 \mathrm{~mA}$. The surface morphology and the elemental distribution of the samples were probed with Scanning
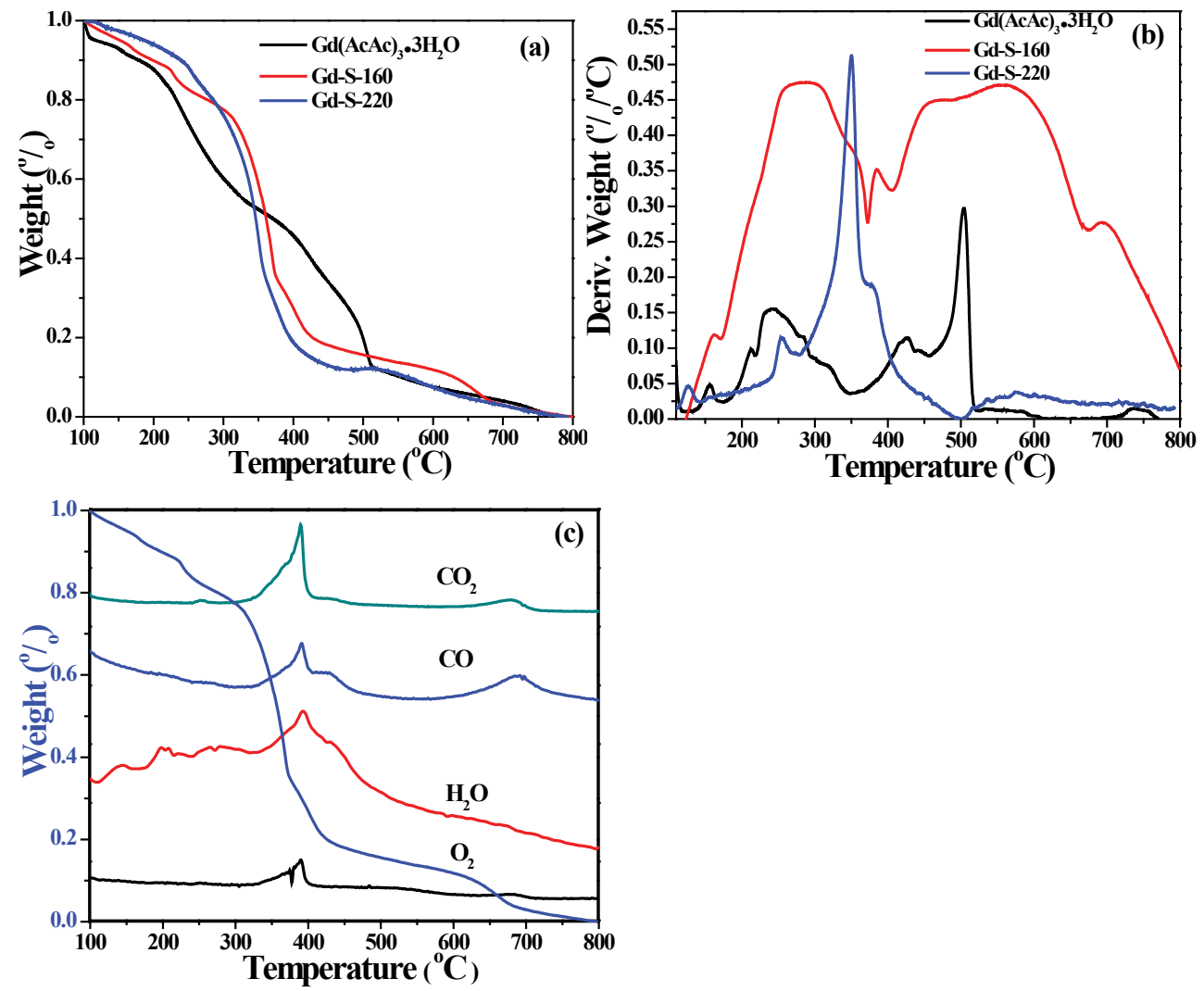

Figure 1: TGA (a) and DTA (b) curves of Gd(AcAc)3·3 $\mathrm{H}_{2} \mathrm{O}$, Gd-S-160, and Gd-S-220, TGA-Ms of Gd-S-160 (c). 
Electron Microscope (SEM, HITACHI S-4300) operated at an acceleration voltage of $5 \mathrm{kV}$. The zeta potential and size distribution of the hybrid particles were measured on Malvern Instruments Zetasizer Nano-ZS90. The Ultraviolet-Visible (UV-vis) spectra were measured with UV6100 double beam spectrophotometer. Room temperature excitation and emission spectra were characterized by F97 spectrofluorometer (Shanghai Lengguang Technology Co., China) with a $150 \mathrm{~W}$ Xe lamp as the excitation source. The temperature-dependent luminescence properties were measured on the fluorescence spectrophotometer (F-4600, HITACHI, Japan), which was equipped with a self-made heating attachment and a computer controlled electric furnace. The luminescence decay curve was obtained from a spectrofluorometer (HORIBA, JOBIN YVON FL3-21) with a $335 \mathrm{~nm}$ pulse laser radiation (nano-LED) as the excitation source, and the pulse width of the laser was $12 \mathrm{~ns}$.

\section{Results and Discussion}

To prepare derivatives of $\mathrm{Gd}(\mathrm{AcAc})_{3} \cdot 3 \mathrm{H}_{2} \mathrm{O}$ by solvothermal method, TGA together with Differential Thermal Analysis (DTA) were performed to determine the decomposition temperature of $\mathrm{Gd}(\mathrm{AcAc})_{3} \cdot 3 \mathrm{H}_{2} \mathrm{O}$. As shown in Figure 1a, the weight losses for all the samples below $150{ }^{\circ} \mathrm{C}$ are assigned to the departure of adsorbed water and comlexed water with gadolinium [15]. Gd(AcAc) $3 \cdot 3 \mathrm{H}_{2} \mathrm{O}$ exhibits complicated decomposition behavior due to more ligands in it. It shows one little and two broad DTA peaks with shoulders between $190-350{ }^{\circ} \mathrm{C}$ and $350-500{ }^{\circ} \mathrm{C}$ (Figure $1 \mathrm{~b}$ ). So it is preferred, $\mathrm{Gd}(\mathrm{A}$ cAc) $33 \mathrm{H}_{2} \mathrm{O}$ derivatives may form around $200{ }^{\circ} \mathrm{C}$ where $\mathrm{Gd}(\mathrm{AcAc})_{3} \cdot 3 \mathrm{H}_{2} \mathrm{O}$ decomposes partially.

Figure 2 shows the FT-IR spectra of the products prepared at around $200{ }^{\circ} \mathrm{C}$. In the spectrum of $\mathrm{Gd}(\mathrm{A}$ cAc) $33 \mathrm{H}_{2} \mathrm{O}$ (Figure $2 \mathrm{a}$ ), the peak at $1602 \mathrm{~cm}^{-1}$ is associated with the enol-keto tautomers of the $\beta$-diketone corresponding to the $\mathrm{C}=\mathrm{O}$ stretching vibration of keto. The peak at $1523 \mathrm{~cm}^{-1}$ is the $\mathrm{C}=\mathrm{C}$ stretching vibration of enol. The peaks at $1391 \mathrm{~cm}^{-1}$ and $1016 \mathrm{~cm}^{-1}$ are the vibrations of $-\mathrm{CH}_{3}[15,16]$. The wide band at about $3300 \mathrm{~cm}^{-1}$ is the vibration of crystallined water of $\mathrm{Gd}(\mathrm{AcAc})_{3} \cdot 3 \mathrm{H}_{2} \mathrm{O}$. The peak at $536 \mathrm{~cm}^{-1}$ is the vibration of a Gd-O stretching absorption. While for the solvothermal samples (Figure $2 \mathrm{~b}-2 \mathrm{~h}$ ), the still appearing peak at $1016 \mathrm{~cm}^{-1}$ relating to the vibration of $-\mathrm{CH}_{3}$ confirms that some acetylacetone groups are still in the final samples although the solvothermal temperature is up to $260{ }^{\circ} \mathrm{C}$ (Figure 2h). The peaks at 1548 and $1441 \mathrm{~cm}^{-1}$ are the $\mathrm{C}=\mathrm{O}$ and $\mathrm{C}=\mathrm{C}$ stretching vibration of $\beta$-diketone which left shift due to the substitution of one or two acetylacetone groups by $\mathrm{OH}$ in the solvothermal process [16]. The peak at $495 \mathrm{~cm}^{-1}$ is the vibration of Gd-O-Gd. The broad peak at $\sim 3415 \mathrm{~cm}^{-1}$ is considered as structural hydroxyl groups

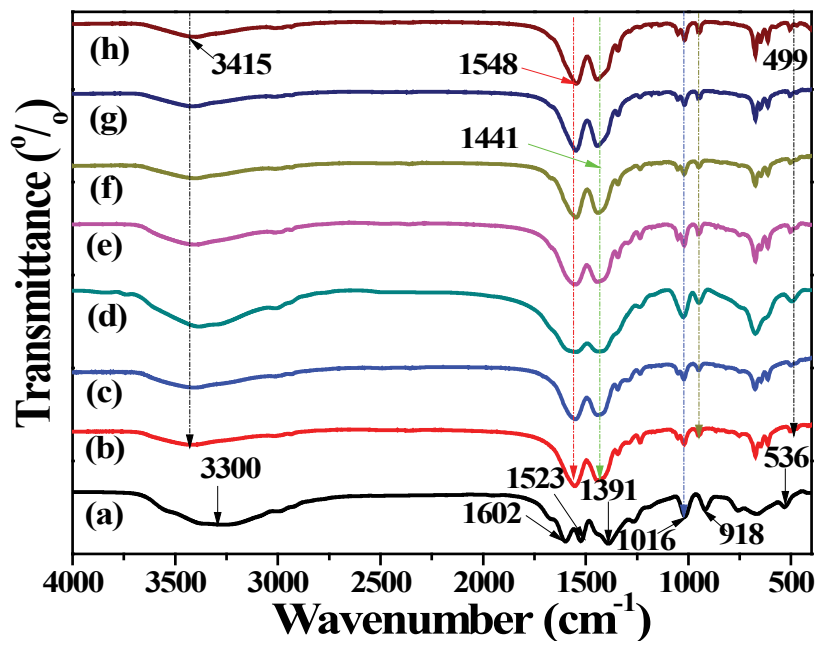

Figure 2: FI-IR spectra of a) $\mathrm{Gd}(\mathrm{AcAc})_{3} \cdot 3 \mathrm{H}_{2} \mathrm{O}$; b) $\mathrm{Gd}-\mathrm{S}-140$; c) Gd-S-160; d) Gd-S-180; e) Gd-S-200; f) Gd-S-220; g) Gd$\mathrm{S}-240$ and h) Ga-S-260 prepared in $2 \mathrm{~h}$.

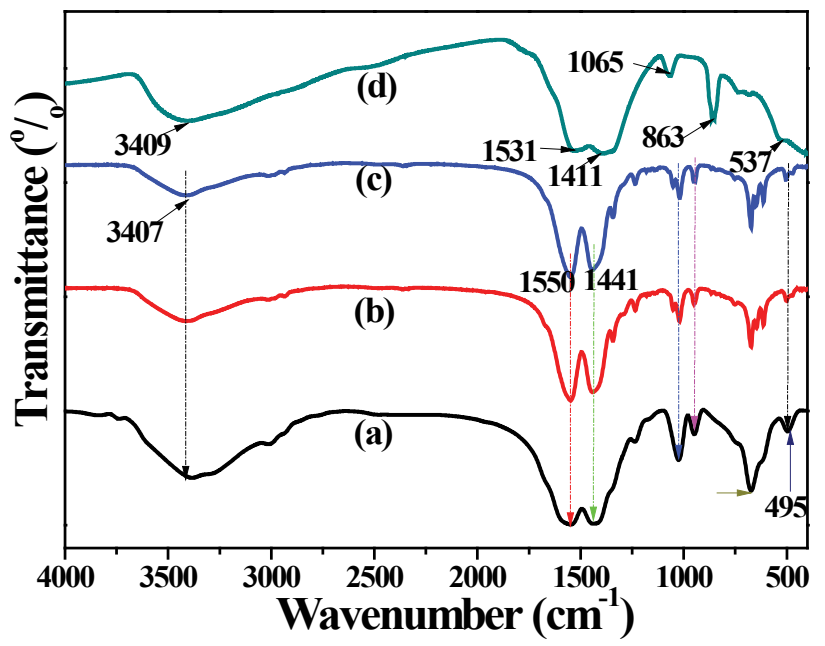

Figure 3: FI-IR spectra of Gd-S-160 prepared in a) 2; b) 4; c) $12 \mathrm{~h}$ and d) Gd-C-160.

connecting Gd. As long then the reaction time from $2 \mathrm{~h}$ to $12 \mathrm{~h}$, the FT-IR spectra of Gd-S-160 (Figure 3) have no obvious change indicating that acetylacetone groups are stable in relative severe solvothermal process. In order to verify that acetylacetone groups are still in the formed samples, Gd-S-160 is calcinated at $450{ }^{\circ} \mathrm{C}$ for $2 \mathrm{~h}$ to give Gd-C-160. Its FTIR spectrum (Figure 3e) shown the peaks at $1548 \mathrm{~cm}^{-1}$ and $1441 \mathrm{~cm}^{-1}$ disappear and bands at $1504 \mathrm{~cm}^{-1}, 1398 \mathrm{~cm}^{-1}, 1096$ and $863 \mathrm{~cm}^{-1}$ due to $v_{\text {as }}$ OC-O, $v_{\mathrm{s}} \mathrm{C}-\mathrm{O}, \mathrm{p}-\mathrm{CO}_{3}{ }^{2-}$ appear, confirming the calcinated sample is $\mathrm{Gd}(\mathrm{OH}) \mathrm{CO}_{3}{ }^{2-}$ or $\left(\mathrm{GdO}_{2}\right) \mathrm{CO}_{3}$ [17]. So it is referred that the solvothermal samples are $\mathrm{Gd}(\mathrm{OH})_{x}(\mathrm{AcAc})_{3-x}$, where $x$ is between 0-3 and AcAc is the acetylacetonate group.

In order to clearly determine the composition of the prepared samples, TGA and DTA of Gd-S-160 and Gd-S-220 were measured (Figure 1). Gd-S-160 and GdS-220 all show one obvious degradation DTA peak be- 
tween $250-400{ }^{\circ} \mathrm{C}$ which corresponds to $\sim 44 \%$ and $32 \%$ weight loss, respectively. If considering the sample is $\mathrm{Gd}(\mathrm{OH})(\mathrm{AcAc})_{2}$ or $\mathrm{Gd}(\mathrm{OH})_{2}(\mathrm{AcAc})$, its corresponding degradation weight loss would be $45 \%$ or $32 \%$. That is the weight losses of Gd-S-160 and Gd-S-220 can well match that of $\mathrm{Gd}(\mathrm{OH})(\mathrm{AcAc})_{2}$ and $\mathrm{Gd}(\mathrm{OH})_{2}(\mathrm{AcAc})$. So the formula of Gd-S-160 and Gd-S-220 are $\mathrm{Gd}(\mathrm{OH})(\mathrm{AcAc})_{2}$ and $\mathrm{Gd}(\mathrm{OH})_{2}(\mathrm{AcAc})$, respectively. Meanwhile the degradation products of Gd-S-160 are measured by TGAMS (Figure 1c). Between $300-400{ }^{\circ} \mathrm{C}$, small molecules including $\mathrm{CO}, \mathrm{CO}_{2}, \mathrm{H}_{2} \mathrm{O}$ are produced confirming the organic groups are preserved in Gd-S-160. In the DTA curve of Gd-S-160 between $450-600{ }^{\circ} \mathrm{C}$, one obvious broad band appear while no degradation occur showing a phase change takes place which may be indexed as the transition of $\mathrm{Gd}$ with 3 complex number to $\mathrm{Gd}_{2} \mathrm{O}_{3}$.

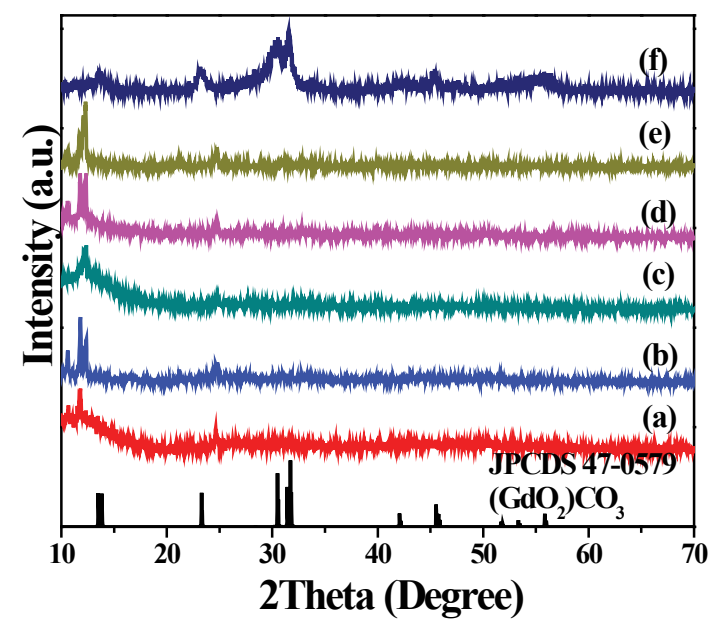

Figure 4: XRD patterns of a) $\mathrm{Gd}(\mathrm{AcAc})_{3} \cdot 3 \mathrm{H}_{2} \mathrm{O}$; b) $\mathrm{Gd}-\mathrm{S}$ 140; c) Gd-S-160; d) Gd-S-180; e) Gd-S-240 and h) Gd-S260 prepared in $2 \mathrm{~h}$, and f) Gd-C-160 prepared in $10 \mathrm{~h}$.

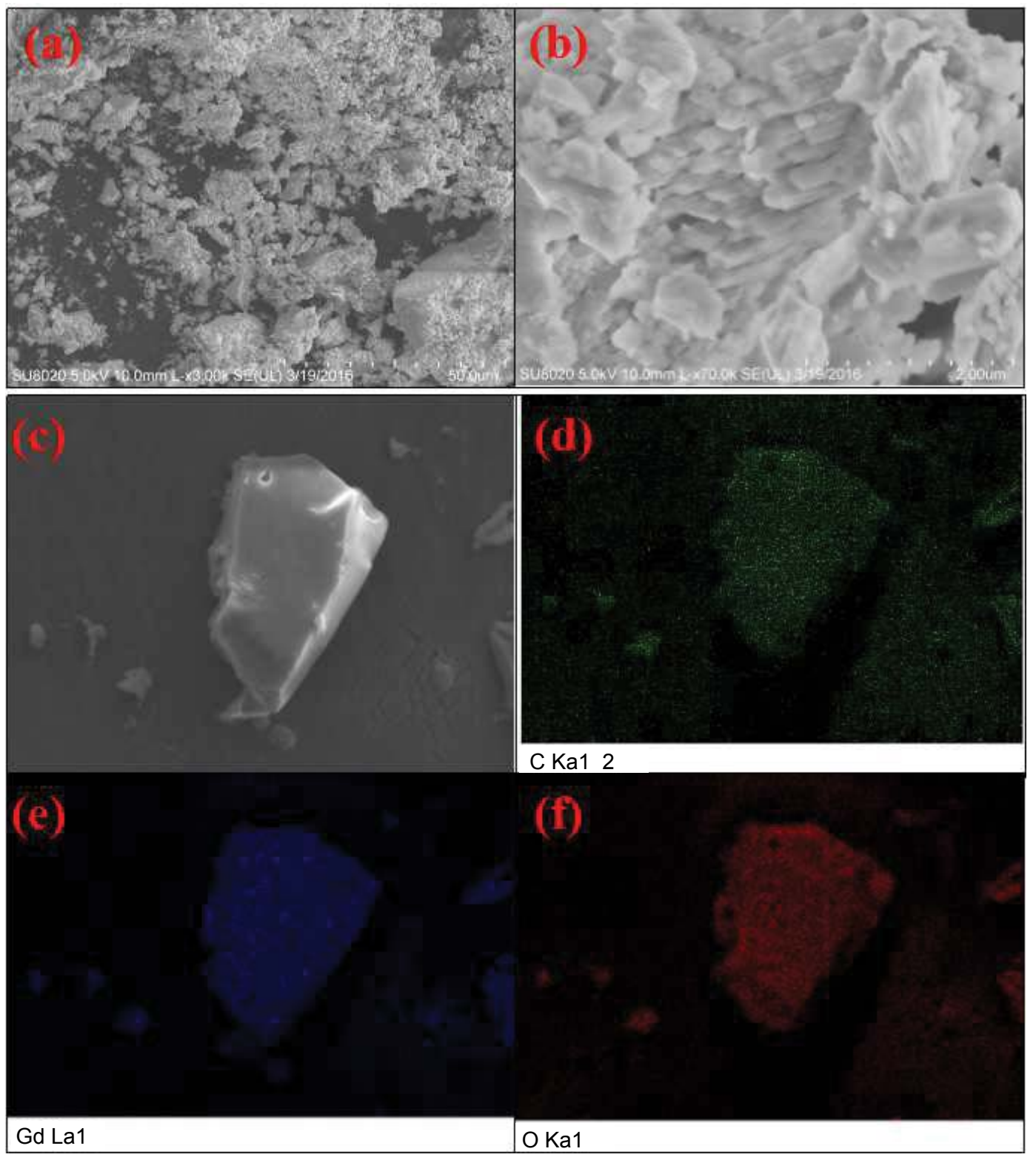

Figure 5: SEM of Gd-S-160 (a,b,c) and the C (d), Gd (e), O (f) dispersion on the particles. 
Figure 4 shows the XRD patterns of Gd-S-T and GdC-160. New peaks appear between $10-15^{\circ}$ compared to $\mathrm{Gd}(\mathrm{AcAc})_{3} \cdot 3 \mathrm{H}_{2} \mathrm{O}$ with the increase of reaction temperature and reaction time. This is due to the substitution of acetylacetone by - $\mathrm{OH}$ with the hydrolysis. Similar phenomenon was reported by hydrolysis of $\mathrm{Gd}(\mathrm{A}$ cAc) $33 \mathrm{H}_{2} \mathrm{O}$ in DMF [18]. The characteristic diffraction peaks of Gd-C-160 can well match that of $\left(\mathrm{GdO}_{2}\right) \mathrm{CO}_{3}$ (JPCDS-47-0579). These result confirms that in diphenyl ether the trace water from structural water of $\mathrm{Gd}(\mathrm{A}$ cAc) $\cdot 3 \mathrm{H}_{2} \mathrm{O}$ can cause $\mathrm{Gd}(\mathrm{AcAc}) \cdot 3 \mathrm{H}_{2} \mathrm{O}$ hydrolyze to form $\mathrm{Gd}(\mathrm{AcAc})_{x}(\mathrm{OH})_{3-x}$ where $x$ is between 0 and 3 .

The SEM and the element dispersions of the prepared Gd-S-160 are shown in Figure 5. In the magnetified photograph (Figure 5b), it can be seen that Gd-S-160 is in rod shape with some irregular particles, the scanning photos show that $\mathrm{C}, \mathrm{Gd}$ and $\mathrm{O}$ uniformly dispersed on the particle confirming the formation of $\mathrm{Gd}(\mathrm{AcAc})_{x}(\mathrm{OH})_{3-x^{*}}$.

The particle sizes of Gd-S-160 and Gd-C-160 were measured by $\mathrm{Z}$ potential and shown in Figure 6 . The particle size of most of Gd-S-160 is $200-400 \mathrm{~nm}$ with little part being about $800 \mathrm{~nm}$. While about $40 \%$ of Gd-C-450 is in the particle size of $200-500 \mathrm{~nm}$, about $50 \%$ in 700 $1200 \mathrm{~nm}$, and about $10 \%$ in $1300-1600 \mathrm{~nm}$. These data show that under suitable solvothermal conditions, the prepared Gd-S-T not only contain organic groups but also have relative small particle size compared to general solvothermal-calcination process. The interesting thing is that no matter Gd-S-T or its calcinated products show positive surface charges. The $\mathrm{Z}$ potential of Gd-S-160 and Gd-C-160 are $42.1 \mathrm{mV}$ and $34.9 \mathrm{mV}$.

Figure 7 shows the UV-v is absorbance of $\mathrm{Gd}(\mathrm{A}$ cAc) $)_{3} \cdot 3 \mathrm{H}_{2} \mathrm{O}$ and Gd-S-T. For $\mathrm{Gd}(\mathrm{AcAc})_{3} \cdot 3 \mathrm{H}_{2} \mathrm{O}$, only one absorbant peak centered at $294 \mathrm{~nm}$ is observed which is attributed to the $\pi-\pi^{*}$ transition of $\beta$-diketonate ligand [5]. Due to the forbidden of electron transition between ligands and $\mathrm{Gd}^{3+}$ which has stable electron arrangement, still only one peak is observed for Gd-S-140, but right shifts to $308 \mathrm{~nm}$ indicating the $\pi-\pi^{*}$ transition of $\beta$-diketonate ligand becomes more easier and the conjugation degree Gd with acetylacetone groups is weakened. For Gd-S-160 and Gd-S-200, two absorbent peaks appear. Except the absorbance at $308 \mathrm{~nm}$, a peak at $275 \mathrm{~nm}$ due to the $\pi-\pi^{*}$ transition of $\beta$-diketonate due to the energy match of ligands after substitution of acetylacetone by $\mathrm{OH}$ groups.

Generally speaking, rare earth organic complexes produce intense emission peaks in visible and near-infrared regions under Ultraviolet (UV) excitation. For obtaining optimum emission spectra, the excitation wavelength for emission measurement is determined by excitation spectrum measurement. Figure $8 \mathrm{a}$ is the excitation fluorescence of Gd-S-T in ultraviolet region under emission

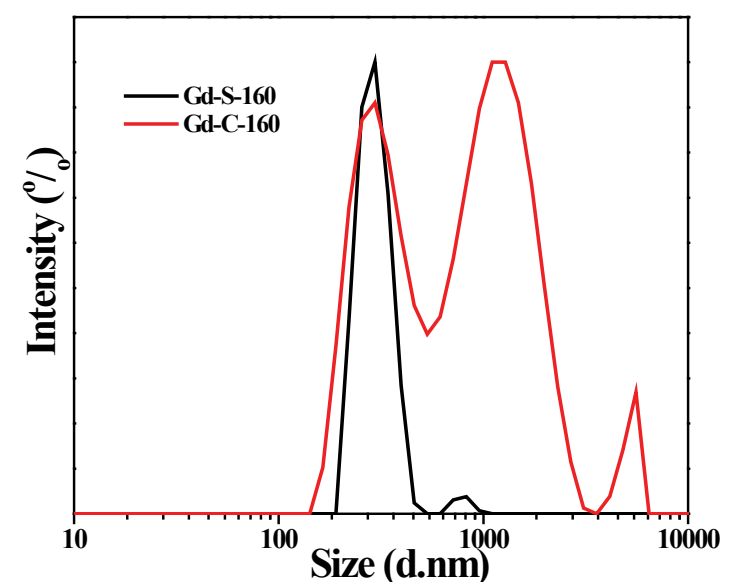

Figure 6: Particle dispersion of Gd-S-160 and Gd-C-160.

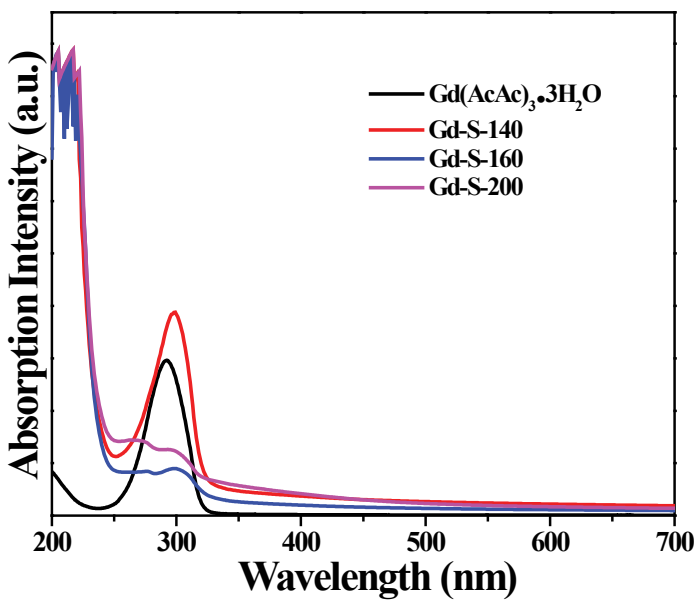

Figure 7: UV-v is absorbance of S-Gd-T.

of $490 \mathrm{~nm}$. $\mathrm{Gd}(\mathrm{AcAc})_{3} \cdot 3 \mathrm{H}_{2} \mathrm{O}$ shows two absorbance, one is between $320-370 \mathrm{~nm}$, the other is between $370-450 \mathrm{~nm}$ attributed to $\pi-\pi^{*}$ and $n-\pi^{*}$ transitions of $\beta$-diketonate ligands [5]. While for Gd-S-140 and Gd-S-160, three peaks are observed which are at 310-350, 350-375 and 375-450 $\mathrm{nm}$. As emission exited under UV irradiation is aimed in the present work, so emission fluorescence is excited by $365 \mathrm{~nm}$ and the spectra are shown in Figure 8b. Gd(AcAc) $3 \cdot 3 \mathrm{H}_{2} \mathrm{O}$ shows one strong emission peak at $520 \mathrm{~nm}$. While the emission of Gd-S-140 and Gd-S-160 left shift indicating that high energy luminescence is produced. Although the acetylacetone groups of $\mathrm{Gd}(\mathrm{AcAc})_{3} \cdot 3 \mathrm{H}_{2} \mathrm{O}$ is more than Gd-S-160, but the emission peak of Gd-S160 is the highest. Just as shown in Figure 9 [19], as the bound ability of -OH to electron is stronger than acetylacetone group, substitution of some of the acetylacetone group by -OH will result in weak interaction of $\mathrm{Gd}^{3+}$ with the left acetylacetone groups of the prepared samples, and thus the controlling ability of acetylacetone group to electron is increased. So the energy between the ground state and exited state in the prepared samples is highered which results in emission of fluorescence with shorter wavelength compared to Gd-acetylacetonate $[7,20]$. As 

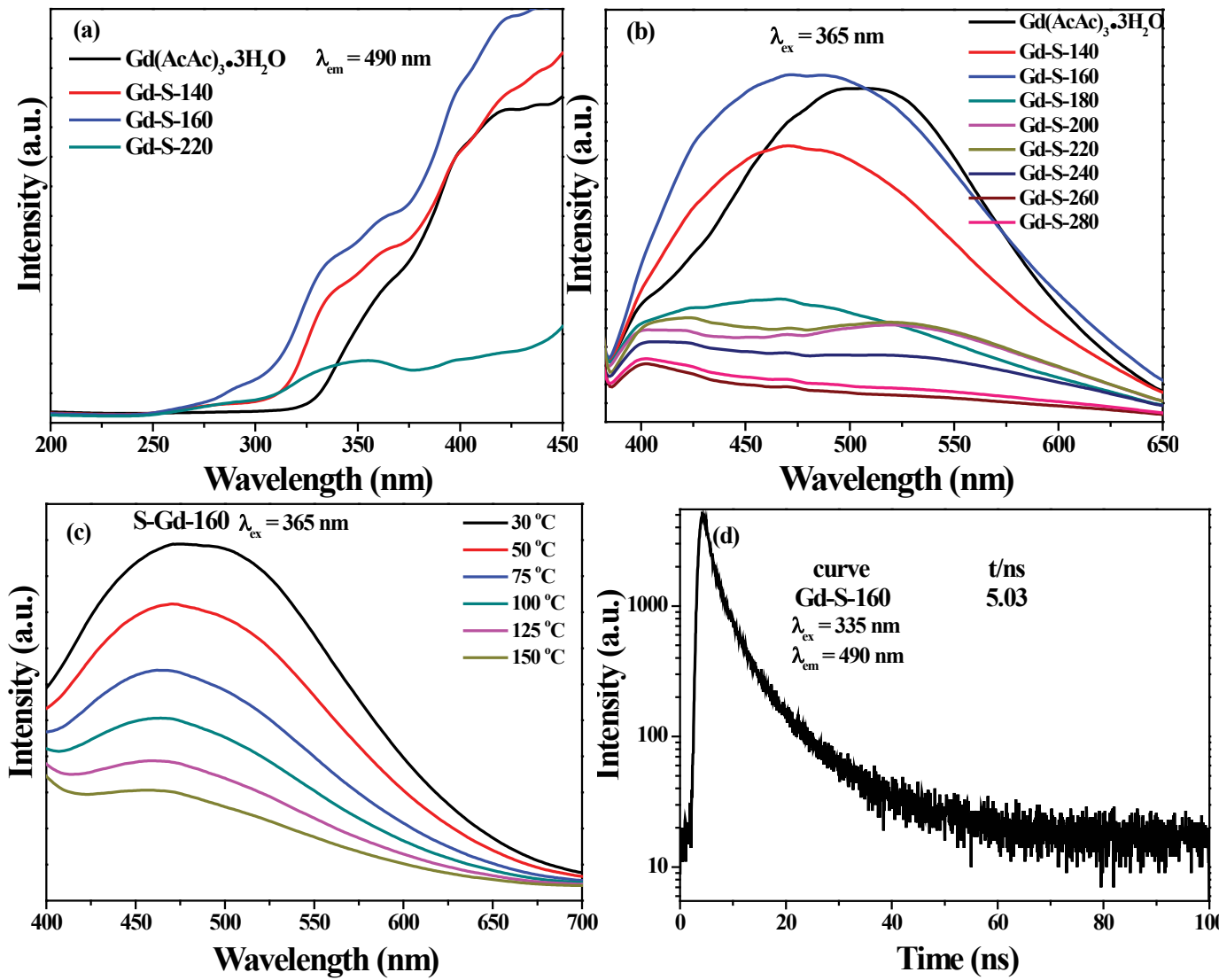

Figure 8: Excitation a) emission; b) fluorescence of Gd-S-T at room temperature and the temperature dependent fluorescence; c) and life time of; d) Gd-S-160.
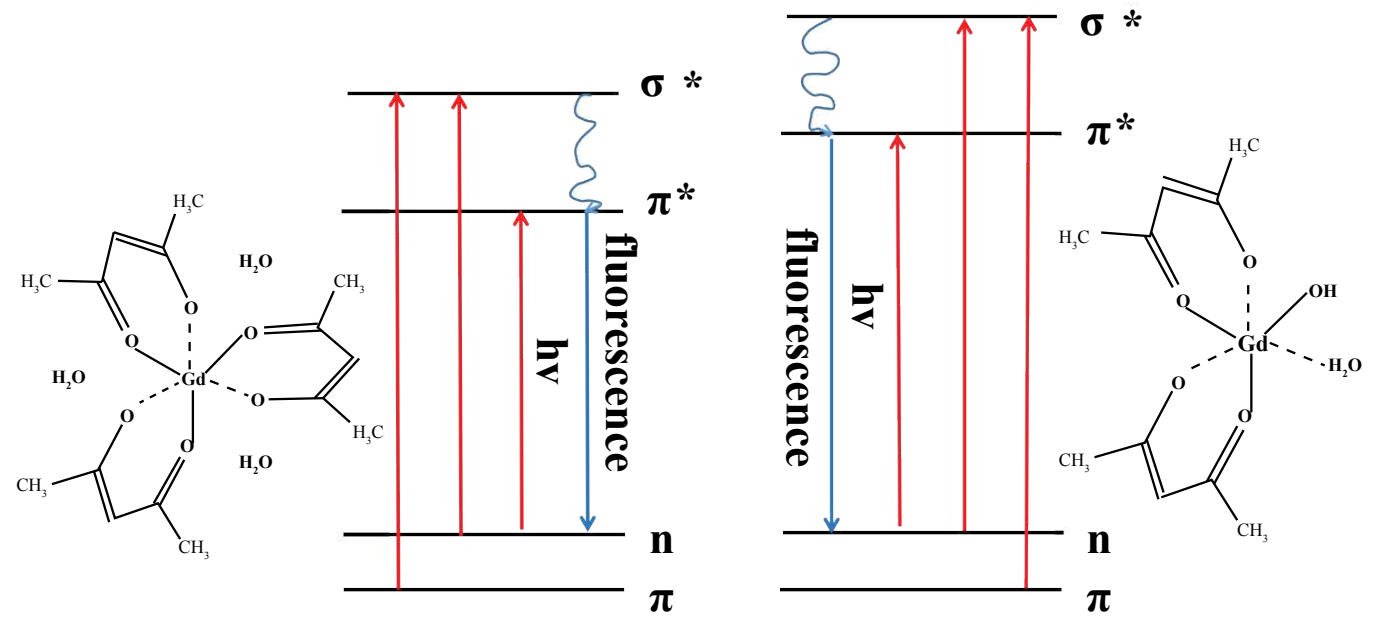

$\boldsymbol{\sigma}$

Figure 9: Energy stage schematic of $\mathrm{Gd}(\mathrm{AcAc})_{3} \cdot 3 \mathrm{H}_{2} \mathrm{O}$ and their products.

the temperature for preparation of Gd-S-T is higher than $200{ }^{\circ} \mathrm{C}$, the emission of the products becomes weak as the amount of acetylacetone groups decrease. Figure $8 \mathrm{c}$ shows the temperature dependence of Gd-S-160. Generally, the fluorescence of organic complex decreases with temperature. At higher temperature, the vibration of electron in acetylacetone increases, so more energy is consumed and the emission strength of the derivatives is decreased, which is confirmed by fluorescent life time measurement. The life time of Gd-S-160 is only 5.03 ns. Short lifetime indicates short time of electrons in existed state and more energy is consumed. With the increase of temperature up to $125^{\circ} \mathrm{C}$, the fluorescence of Gd-S160 decreases sharply and left shifts due to the weakened 


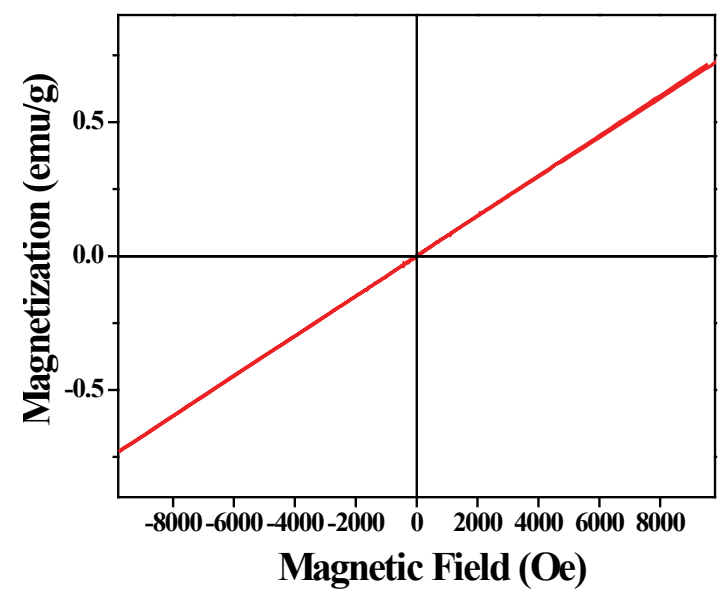

Figure 10: Magnetization of Gd-S-160.

interaction strength between acetylacetone and Gd with temperature and the controlling ability of acetylacetonate to electrons is increased. Therefore the obtained samples are not temperature stable enough.

Figure 10 shows M-H curve of Gd-S-160 measured at $300 \mathrm{~K}$. The magnetization of Gd-S-160 increases linearly with the testing magnetic strength increase. This phenomenon is due to the magnetizing current for $\mathrm{Ms}$ Measurement of Gd-S-160 is too high. In other words, Gd-S-160 is relatively easy magnetization. As the applied magnetic field is $8000 \mathrm{Oe}$, the magnetization of Gd-S-160 is $0.72797 \mathrm{em} \mu / \mathrm{g}$ confirming the produced samples can response to external magnetic field.

\section{Conclusions}

Gadolinium (III) acetylacetonate was treated by solvothermal method to produce Gadolinium (III) acetylacetonate derivatives. The composition, fluorescence, and magnetic properties of the products are studied in detail. The fluorescence measurement showed the products possessed higher energy emission and excitation fluorescence compared to Gadolinium (III) acetylacetonate. Also the product produced at $160{ }^{\circ} \mathrm{C}$ possessed higher fluorescent intensity. Based on the fluorescent study the fluorescent emission mechanism is proposed. The magnetic measurement indicated the products were paramagnetic materials. So the products were hydrophobic materials with magnetic and fluorescent properties.

\section{Acknowledgements}

The work was jointly supported by the Fundamental Research Funds for the Central Universities (2-9-2016087).

\section{References}

1. Scotognella F, Meinardi F, Ottonelli M, Raimondo L, Tubino $R$ (2009) Photophysical analysis of the organic complex $\left[\mathrm{Eu}\left(\mathrm{C}_{12} \mathrm{H}_{8} \mathrm{~N}_{2}\right)_{2}\right]\left(\mathrm{NO}_{3}\right)_{3}$. J Lumin 129: 746-750.
2. Zhang W, He W, Guo X, Chen Y, Wu L, et al. (2015) Synthesis and luminescence properties of 1,3,4-oxadiazole acetamide derivatives and their rare earth complexes. $J$ Alloys Compd 620: 383-389.

3. Zhang YY, Shen LF, Pun EYB, Chen BJ, Lin H (2013) Multi-color fluorescence in rare earth acetylacetonate hydrate doped poly methyl methacrylate. Opt Commun 311: 111-116.

4. Lapaev DV, Nikiforov VG, Safiullin GM, Lobkov VS, Knyazev AA, et al. (2016) Changes in luminescent properties of vitrified films of terbium(III) $\beta$-diketonate complex upon UV laser irradiation. J Lumin 175: 106-112.

5. Thejo Kalyani N, Dhoble SJ, Pode RB (2012) Enhancement of photoluminescence in various $\mathrm{Eu}_{\mathrm{x}} \mathrm{Re}_{(1-x)} \mathrm{TTA}_{3}$ Phen ( $\mathrm{Re}=$ $\mathrm{Y}, \mathrm{Tb})$ complexes molecularly doped in PMMA. Indian $\mathrm{J}$ Phys 86: 613-618.

6. Zhang RJ, Yang KZ, Yu AC, Zhao XS (2000) Fluorescence lifetime and energy transfer of rare earth $\beta$-diketone complexes in organized molecular films. Thin Solid Films 363: 275-278.

7. Penzkofer A (2013) Phosphorescence quantum yield determination with time-gated fluorimeter and $\mathrm{Tb}$ (III)-acetylacetonate as luminescence reference. Chem Phys 415: 173-178.

8. Sun WB, Han BL, Lin PH, Li HF, Chen P, et al. (2013) Series of dinuclear and tetranuclear lanthanide clusters encapsulated by salen-type and beta-diketionate ligands: single-molecule magnet and fluorescence properties. Dalton Trans 42: 13397-13403.

9. Gao HL, Jiang L, Liu S, Shen HY, Wang WM, et al. (2016) Multiple magnetic relaxation processes, magnetocaloric effect and fluorescence properties of rhombus-shaped tetranuclear rare earth complexes. Dalton Trans 45: 253-264.

10. Křenek T, Kovářík T, Pola $M$, Jakubec I, Bezdička $P$, et al. (2013) Enhancement of thermal stability of silver(I) acetylacetonate by platinum(II) acetylacetonate. Thermochim Acta 554: 1-7.

11. Chin SF, Pang SC, Tan CH (2011) Green synthesis of magnetite nanoparticles (via thermal decomposition method) with controllable size and shape. J Mater Environ Sci 2: 299-302.

12. Sun S, Murray CB, Weller D, Folks L, Moser A (2000) Monodisperse FePt nanoparticles and ferromagnetic FePt nanocrystal superlattices. Science 287: 1989-1992.

13. Tangwatanakul W, Chokprasombat K, Sirisathitkul C, Jantaratana P, Sirisathitkul Y (2016) Magnetic phase transition of annealed FePt based nanoparticles synthesized by using $\mathrm{Fe}(\beta \text {-diketonate })_{3}$. J Alloys Compd 654: 234-239.

14. Paek J, Lee CH, Choi J, Choi SY, Kim A, et al. (2007) Gadolinium Oxide Nanoring and Nanoplate: Anisotropic Shape Control. Cryst Growth Des 7: 1378-1380.

15. Molina R, Poncelet G (1999) a-Alumina-Supported Nickel Catalysts Prepared with Nickel Acetylacetonate. 2. A Study of the Thermolysis of the Metal Precursor. J Phys Chem B 103: 11290-11296.

16. Shen C, Shaw LL (2009) FTIR analysis of the hydrolysis rate in the sol-gel formation of gadolinia-doped ceria with acetylacetonate precursors. J Sol Gel Sci Technol 53: 571577 . 
17. Liu Y, Liu G, Wang J, Dong X, Yu W (2015) Multicolor photoluminescence and energy transfer properties of dysprosium and europium-doped $\mathrm{Gd}_{2} \mathrm{O}_{3}$ phosphors. J Alloys Compd 649: 96-103.

18. Shaw LL, Shen C, Thomas EL (2009) Synthesis of gadolinia-doped ceria gels and powders from acetylacetonate precursors. J Sol Gel Sci Technol 53: 1-11.

19. Agondanou JH, Nicolis I, Curis E, Purans J, Spyroulias
GA, et al. (2007) Gadolinium Acetylacetonate Tetraphenyl Monoporphyrinate Complex and Some of Its Derivatives: EXAFS Study and Molecular Dynamics Simulation. Inorg Chem 46: 6871-6879.

20. Gavrikov AV, Koroteev PS, Dobrokhotova ZV, llyukhin AB, Efimov NN, et al. (2015) Novel heterometallic polymeric lanthanide acetylacetonates with bridging cymantrenecarboxylate groups-synthesis, magnetism and thermolysis. Polyhedron 102: 48-59. 